\title{
Exploring the Limit of Dislocation Based Plasticity in Nanostructured Metals
}

\author{
Hughes, D. A.; Hansen, Niels
}

Published in:

Physical Review Letters

Link to article, DOI:

10.1103/PhysRevLett.112.135504

Publication date:

2014

Document Version

Publisher's PDF, also known as Version of record

Link back to DTU Orbit

Citation (APA):

Hughes, D. A., \& Hansen, N. (2014). Exploring the Limit of Dislocation Based Plasticity in Nanostructured Metals. Physical Review Letters, 112(13), [135504]. https://doi.org/10.1103/PhysRevLett.112.135504

\section{General rights}

Copyright and moral rights for the publications made accessible in the public portal are retained by the authors and/or other copyright owners and it is a condition of accessing publications that users recognise and abide by the legal requirements associated with these rights.

- Users may download and print one copy of any publication from the public portal for the purpose of private study or research.

- You may not further distribute the material or use it for any profit-making activity or commercial gain

- You may freely distribute the URL identifying the publication in the public portal

If you believe that this document breaches copyright please contact us providing details, and we will remove access to the work immediately and investigate your claim. 


\title{
Exploring the Limit of Dislocation Based Plasticity in Nanostructured Metals
}

\author{
D. A. Hughes ${ }^{1, *}$ and N. Hansen ${ }^{2}$ \\ ${ }^{1}$ Sandia National Laboratories, Livermore, California 94551-0969, USA \\ ${ }^{2}$ Department of Wind Energy, Technical University of Denmark, Ris $\phi$ Campus, Roskilde DK4000, Denmark
}

(Received 16 August 2013; published 4 April 2014)

\begin{abstract}
A twofold decrease to an unexplored scale of $5 \mathrm{~nm}$ was produced in $\mathrm{Cu}$ by applying a large sliding load in liquid nitrogen. Statistical and universal scaling analyses of deformation induced high angle boundaries, dislocation boundaries, and individual dislocations observed by high resolution electron microscopy reveal that dislocation processes still dominate. Dislocation based plasticity continues far below the transition suggested by experiment and molecular dynamics simulations, with a limit below $5 \mathrm{~nm}$. Very high strength metals may emerge based on this enhanced structural refinement.
\end{abstract}

DOI: 10.1103/PhysRevLett.112.135504

PACS numbers: 62.25.-g, 61.46.Hk, 61.72.Ff, 62.20.F-

Dislocation processes govern the deformation regime in which the quintessential attributes of strength and plasticity of metals and alloys can be tuned. Structural refinement generally increases strength. Thus, to simultaneously achieve the finest structural scale and retain the dominance of dislocation processes is essential.

Previously, we produced nanostructured $\mathrm{Cu}$ by plastic deformation to ultrahigh strains utilizing large sliding loads [1]. The minimum average boundary spacing produced was $12 \mathrm{~nm}$. That sample strongly exhibited the characteristics of dislocation based processes and clear evidence of their collective behavior. Experiments using different deformation methods including high pressure torsion [2], grinding [3], and repetitive rolling and folding [4] also show dislocation processes at similar size scales. A change from these dislocation processes to grain boundary sliding which leads to softening below 10 to $20 \mathrm{~nm}$ may be anticipated based on experiment and molecular dynamics (MD) simulations [5-7] To elucidate the deformation mechanisms operating below this range, we designed a new sliding experiment with the goal of reducing the size scale significantly below $10 \mathrm{~nm}$. Statistical mechanics analyses using high resolution electron microscopy (HREM) of the resulting nanostructure was applied to unambiguously extract information on structural evolution and the deformation mechanisms.

To achieve the finest scale and isolate size effects on structure evolution requires a critical balance in deformation parameters including strain level, strain rate, temperature, and alloying consistent with our previous work [1]. During deformation dislocation based processes, e.g., glide, as well as possibly twinning, can reduce grain size but extreme strains, $>150$, are required to reach the nanometer scale. (The maximum strain in conventional rolling is from 5 to 10.) Such extreme strains were achieved in our new apparatus which enabled a fourfold increase to $55 \mathrm{MPa}$ in the normal pressure between the $\mathrm{Cu}$ sample and the steel platen. A large area, $645 \mathrm{~mm}^{2}$, of this uniform pressure was maintained as the $\mathrm{Cu}$ sample was slid once over a specially ground steel platen thereby inducing waves of shear strain in the near surface layers. Depth and degree of deformation increase with increasing normal pressure. As before, a very slow sliding rate during the experiment kept the sample strain rate low: $\sim 1 \mathrm{~s}^{-1}$, akin to laboratory rolling, to inhibit twinning which is more prominent at very high strain rates.

Grain and structural coarsening, driven by increasing total grain boundary and dislocation energies which aid thermally activated recovery and recrystallization processes, are a constant threat to refinement. Recrystallization and grain growth can occur in highly deformed pure copper at room temperature $[1,8]$. Two approaches stabilized the fine structure. The temperature was lowered by immersing the apparatus in liquid nitrogen. Copper was prealloyed with $0.5 \mathrm{wt} . \% \mathrm{Fe}$. Additional $\mathrm{Fe}$ (about $+1 \%$ ) diffuses into the near surface regions from the steel platen during sliding. A scanning transmission electron analysis showed the Fe to be finely distributed. No particles were observed and the $\mathrm{Fe}$ is thought to be in solution. Both Fe sources are needed as grain growth and recrystallization were observed following sliding in liquid nitrogen for solutions below 0.5 wt. $\% \mathrm{Fe}$; structural stability was observed above 1 wt.\% Fe.

Unambiguous examination of grain sizes far below $10 \mathrm{~nm}$ was achieved by adapting HREM. Large areas of resolved contiguous grains are difficult to observe but were required for a scaling analysis of microstructure evolution. Sliding deformation naturally produces preferred crystal orientations accessible to HREM analysis in the sample plane observed. This plane contains the shear plane normal and shear directions. Areas with $\langle 110\rangle$ foil normals variously rotated about the radial direction are representative of the structure. Measurements of boundary spacing, crystal orientation, deformation texture, and calculation of boundary misorientation angles provided the necessary parameters for the analysis. 
In each crystallite, lattice fringes were indexed based on both their intersection angles and spacing to determine the beam direction and [002]. Two to three indexed fringes were required depending on their identity. Care was taken to avoid confusing fine Moire fringes with lattice fringes especially for special overlapping orientations [9]. Threedimensional crystal orientations were determined by using the beam direction and substituting the angle of the [002] fringe to the reference sample axes into the orientation and misorientation (disorientation) calculations developed for Kikuchi patterns [10].

Within contiguous regions, boundaries between differently oriented crystals or grains were identified and marked as the locations where lattice fringe(s) diverge by $\geq 5^{\circ}$. We have previously classified these extended boundaries as geometrically necessary boundaries (GNB). For boundaries inclined to the foil, the boundary location was taken at the center of the overlap. Adjacent crystallites were identified to determine boundary misorientations and spacings, $D^{\mathrm{GNB}}$. Spacing was measured as an intercept length perpendicular to both the sliding direction and surface, i.e., perpendicular to the lamellar structure that also includes boundaries forming a more equiaxed structure. This structure (Fig. 1) is like that observed in the previous sliding experiment, albeit with a lower aspect ratio.

Rigorous measurements show that the new experiments successfully reduced the average size scale for the boundary spacing by more than a factor of 2 . The new average, $D_{\mathrm{av}}^{\mathrm{GNB}}$, is $5.2 \mathrm{~nm}$ with a narrow distribution, e.g., $60 \%$ of the spacings are below $5 \mathrm{~nm}$ (Fig. 2). Few spacings, 4\%, range from 10 to $20 \mathrm{~nm}$. The boundaries have a large average misorientation of $39^{\circ}$ and large fraction, $70 \%$, of high angle boundaries $\geq 15^{\circ}$ Formation of a preferred crystallographic deformation texture is confirmed by the imaging of large contiguous regions within the [110] radial fiber in the HREM images. The majority of these orientations cluster near the ideal $A_{1}^{*}(111)[2 \overline{1} \overline{1}], A_{2}^{*}(\overline{1} 11)[211]$, and C $\{001\}\langle 1 \overline{1} 0\rangle$ shear components with the $\mathrm{C}$ the most frequent. Note $(h k l)[u v w]$ refer to the shear plane and shear direction. Other components could not be imaged in this cross section.

The distribution of minimum misorientation angles, i.e., the disorientation, is shown in Fig. 3. The distribution shape reveals a small peak at low to medium angles followed by a second high angle peak like the distributions found for deformation modes with larger microstructural size scales. The peak at high angles is associated with texture formation [11]. Low angle boundaries created during deformation are still identifiable as dislocation boundaries. The low and high angle boundaries are interlinked within the structure. A few deformation twins were identified by their paired $\Sigma 3$ boundaries: $60^{\circ}$ with a $\langle 111\rangle$ rotation axis. These twins represent only $4 \%$ of the measured crystallites and $8 \%$ of the GNBs. The paucity of twins is representative of the structure as HREM images are biased to reveal deformation twins in the observed cross section.

The peak in the misorientation distribution at $60^{\circ}$ is primarily a result of texture formation about $\mathrm{C}, A_{1}^{*}$, and $A_{2}^{*}$ and the measurement along the radial [110] fiber. Adjacent orientations along a [110] fiber that are rotated from $60.7^{\circ}$ to $90^{\circ}$ about [110] from each other have misorientations

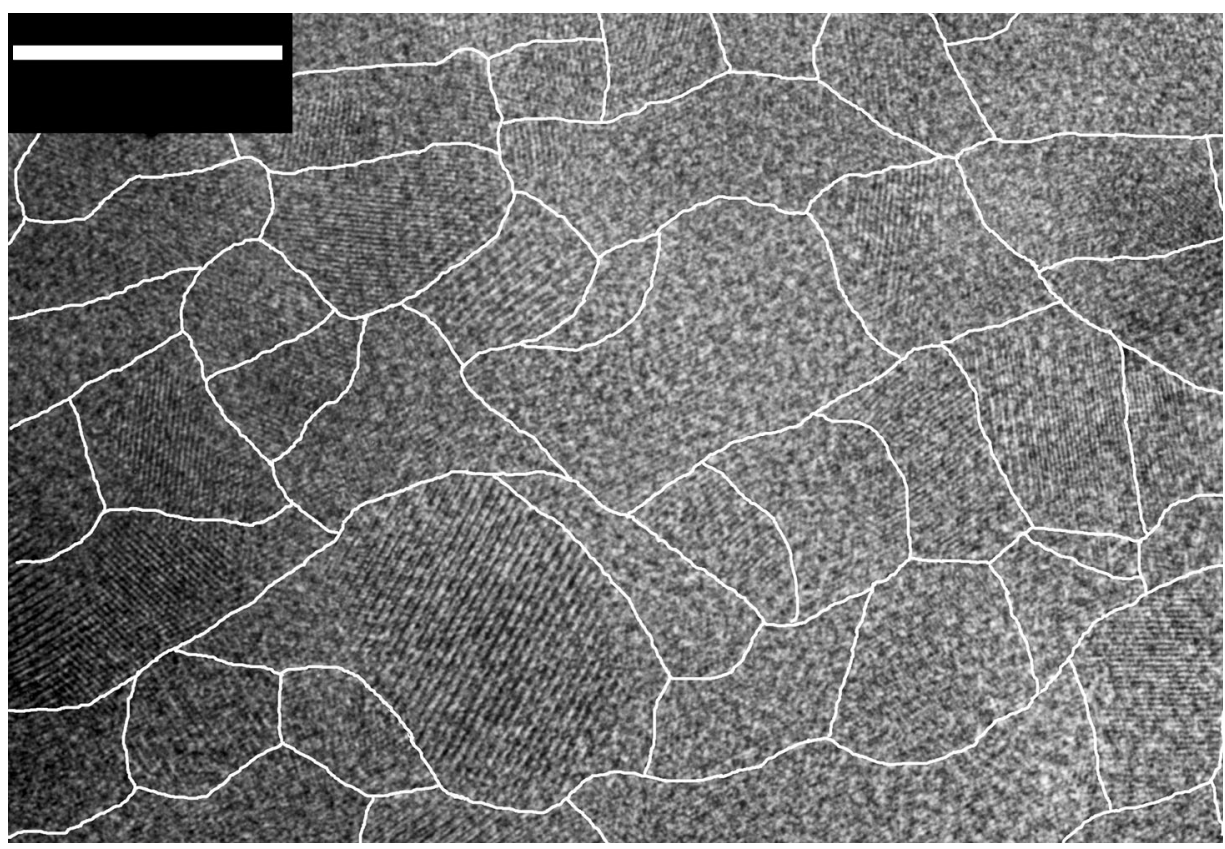

FIG. 1. Nanostructure produced by friction viewed in cross section by HREM. Tracings of the boundaries are overlaid in white. The horizon is parallel to the shear direction and the vertical to the shear plane normal. The scale marker is $10 \mathrm{~nm}$. 


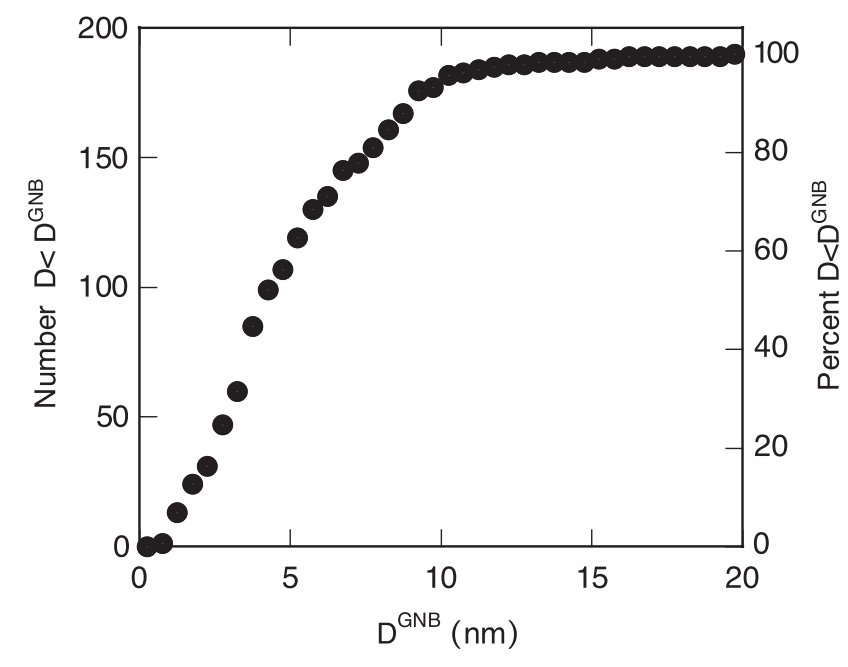

FIG. 2. Cumulative distribution of $D^{\mathrm{GNB}}$ boundary intercept spacings.

near $60^{\circ}$. Their differences are revealed by their rotation axes.

Together with the grain structure formed by the boundaries, some dislocations are observed within grain interiors, Fig. 4, and attached to low and high angle boundaries (see also [12]). These dislocations are primarily $60^{\circ}$ glide dislocations dissociated into Shockley partials separated by short stacking faults. These dislocations have narrowed stacking faults consistent with a stress screened low energy structure [13]. Stacking faults dividing grains or attached to just a single partial dislocation were rare. Only dislocations with an edge component in the viewing plane are visible by HREM, which provides a lower bound for the dislocation density.

The quantified structure reveals an evolution during deformation towards ever finer nanocrystals separated by boundaries. This evolution takes place via structural subdivision from a structure dominated by low angle dislocation boundaries at low and medium strain, to one with a

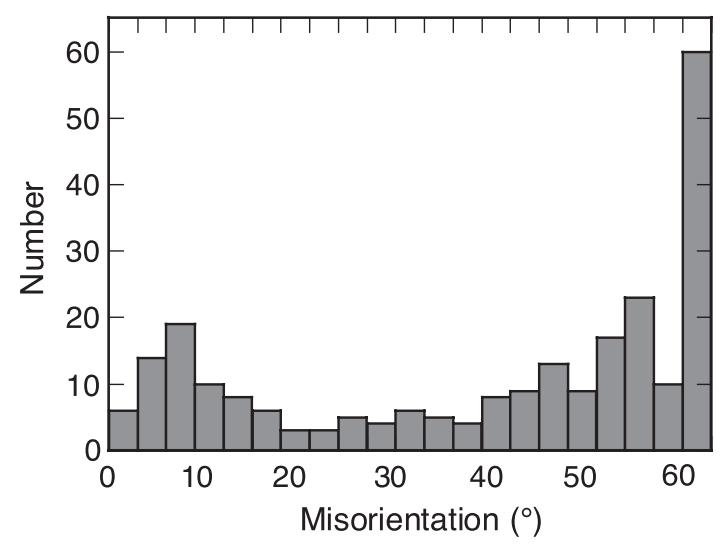

FIG. 3. Distribution of boundary misorientations for $D_{\mathrm{av}}^{\mathrm{GNB}}=$ $5.2 \mathrm{~nm}$ in $\mathrm{Cu}$ deformed by friction. large fraction, $70 \%$, of high angle boundaries at high and ultrahigh strains.

This new structure can be quantitatively compared with structures at lower strains and coarser structures using a scaling analysis. In monotonic deformation the probability distributions $p\left(D^{\mathrm{GNB}}, D_{\mathrm{av}}^{\mathrm{GNB}}\right)$ of individual perpendicular spacings, $D^{\mathrm{GNB}}$, between the boundaries at a given strain level or condition, can be scaled by their average spacing, $D_{\mathrm{av}}^{\mathrm{GNB}}$, into a single universal distribution $[1,14]$. Thus, $p\left(D^{\mathrm{GNB}}, D_{\mathrm{av}}^{\mathrm{GNB}}\right)=\left(D_{\mathrm{av}}^{\mathrm{GNB}}\right)^{-1} f\left(D^{\mathrm{GNB}} / D_{\mathrm{av}}^{\mathrm{GNB}}\right)$ is a simple function of the average spacing. Following this normalization, the new distribution collapses into the same universal distribution as before Fig. 5. Remarkably, this universal behavior now applies to the next lower order of magnitude in average spacing resulting in an extraordinary range of four orders.

Evidence for the significant activity of dislocation based mechanisms is provided by several observations: substantial structural refinement as high angle and dislocation boundaries are created subdividing the structure, the presence of a shear texture, and a high dislocation density stored in low and medium angle boundaries as well as between and attached to boundaries. Universal scaling indicates a continuity in the underlying processes that refine the microstructure thereby creating and removing boundaries from low to high strain, including dislocation slip processes.

Any significant role of other mechanisms is ruled out by key evidence. Previous work provides contrasting suggestions from the severely reduced activity of dislocations to a transition to other dominating deformation mechanisms at diminished structural size. These mechanisms include deformation twinning, grain boundary sliding, and stress induced grain growth via grain boundary sliding $[5,6]$. We see complete glide dislocations below 15 to $10 \mathrm{~nm}$, while MD simulations do not [6,7]. We rarely see isolated Shockley partial dislocations or stacking faults spanning grain boundaries; MD observes many [6,7]. We see few deformation twins, MD predicts many $[6,7]$. Our continuous structural refinement to $5 \mathrm{~nm}$ is the opposite of stress-induced grain growth found in MD [6]. Our deformation texture indicates dislocation glide not grain boundary sliding which randomizes the texture.

The presence of high angle boundaries and a large driving pressure due to the high stored energy of the deformed structure suggests increasing structural instability, which is not observed. Thus, something must reduce the boundary mobility. The presence of an Fe solute may be one cause for the mobility reduction. The presence of dislocations attached to boundaries and the interlinkage of low and high angle boundaries may be additional factors that inhibit structural coarsening.

The observed structural refinement during deformation has been extended to an average boundary spacing of $5 \mathrm{~nm}$ that includes $70 \%$ high angle boundaries. The continuous 


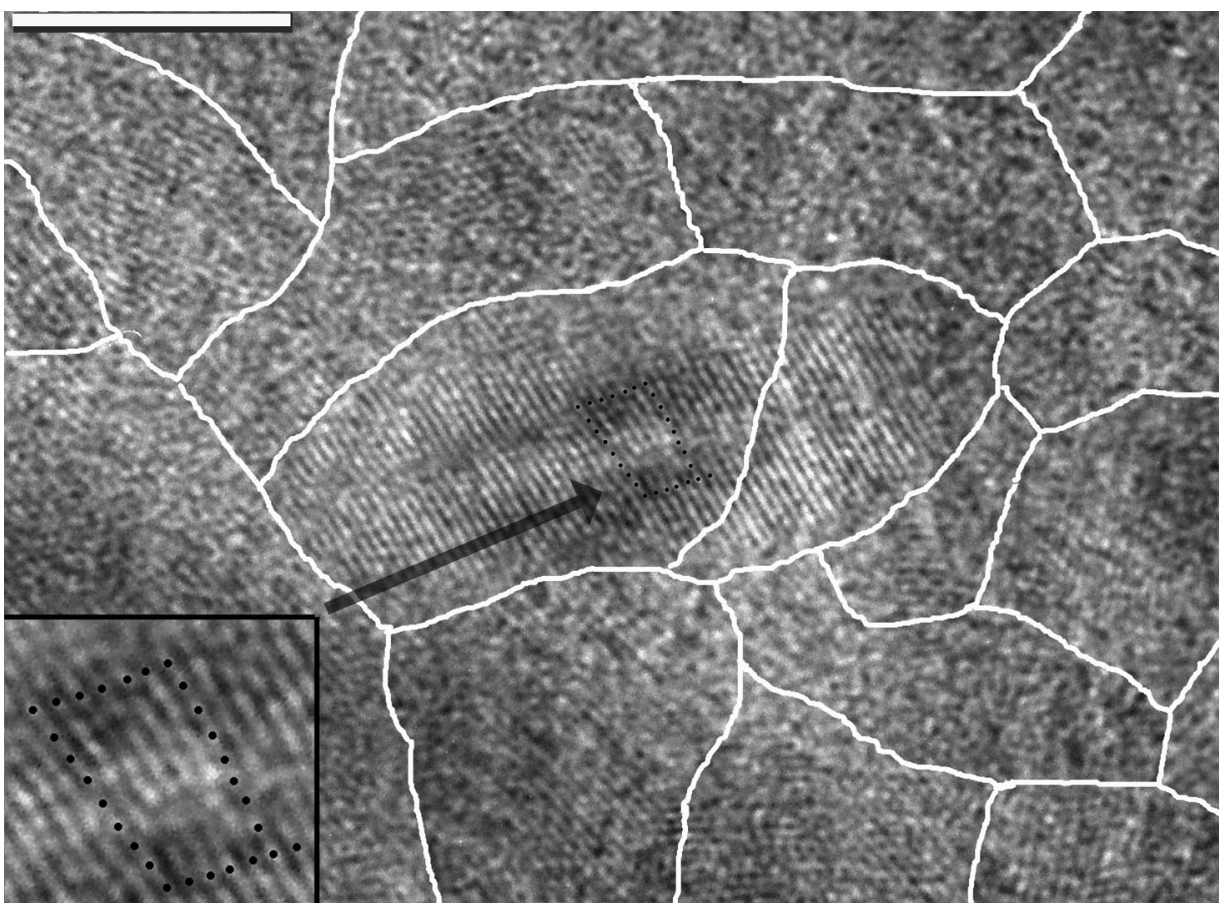

FIG. 4. Nanostructure produced by friction viewed in cross section by HREM. The enlarged inset shows a glide dislocation marked with a Burgers circuit of black dots; the arrow indicates its position in the central nanocrystal. Tracings of the boundaries are overlaid in white. The horizon is parallel to the shear direction and the vertical to the shear plane normal. The scale marker is $5 \mathrm{~nm}$.

structural subdivision and the high concentration of dislocations observed shows no physical limit to dislocation based plasticity. The fine structure of the sliding layer and the expected high strength suggest potential applications, e.g., as a hard wear resistant surface on small components.

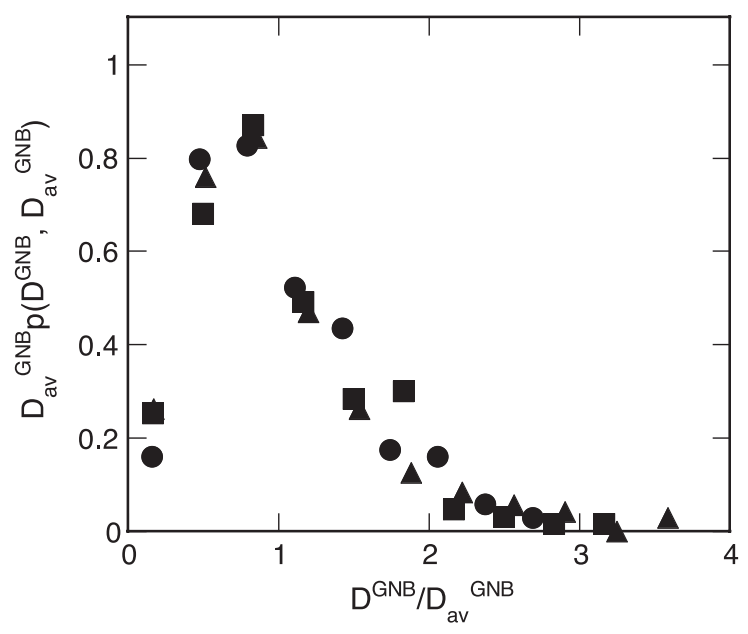

FIG. 5. Probability distribution of nanometer scale boundary spacings produced by friction normalized by the average spacing compared to distributions at a larger scale in rolled nickel. Friction, solid circles: $D_{\mathrm{av}}^{\mathrm{GNB}}=5.2 \mathrm{~nm}$; triangles: $\mathrm{Ni} 70 \%$ reduction, $\quad D_{\mathrm{av}}^{\mathrm{GNB}}=280 \mathrm{~nm}$; squares: $\mathrm{Ni} 98 \%$ reduction, $D_{\mathrm{av}}^{\mathrm{GNB}}=133 \mathrm{~nm}$. Rolling data from Ref. [11].
D. A. H. and N. H. acknowledge and thank J. Korellis and D. Dawson for the new design of the sliding apparatus, P. Nielsen for casting the $\mathrm{Cu} 0.5 \% \mathrm{Fe}$, G. Christensen for preparing large area HREM foils, and D. Yaney and A. Godfrey for discussion. The work performed while D. A. H. was at Sandia was supported by the Office of Basic Energy Sciences, Division of Materials and Engineering Sciences, U. S. Department of Energy. Sandia National Laboratories is a multiprogram laboratory managed and operated by Sandia Corporation, a wholly owned subsidiary of Lockheed Martin Corporation, for the U.S. Department of Energy's National Nuclear Security Administration under Contract No. DE-AC04-94AL85000. N.H. acknowledges the Danish National Research Foundation Grant No. DNR F86-5.

*darcyahughes@gmail.com

[1] D. A. Hughes and N. Hansen, Phys. Rev. Lett. 87, 135503 (2001).

[2] H. W. Zhang, X. Huang, and N. Hansen, Acta Mater. 56, 5451 (2008).

[3] T. H. Fang, W. Li, N. R. Tao, and K. Lu, Science, 331, 1587 (2011).

[4] G. Wilde and H. Rösner, J. Mater. Sci. 42, 1772 (2007).

[5] H. Hahn, P. Mondal, and K. A. Padmanabhan, Nanostruct. Mater. 9, 603 (1997).

[6] D. Wolf, V. Yamakov, S. R. Phillpot, A. Mukherjee, and H. Gleiter, Acta Mater. 53, 1 (2005).

[7] J. Schiotz, Scr. Mater. 51, 837 (2004). 
[8] J. Gil-Sevillano, P. van Houtte, and E. Aernoudt, Prog. Mater. Sci. 25, 69 (1980).

[9] C. Rentenberger, T. Waitz, and H. P. Karnthaler, Scr. Mater. 51, 789 (2004).

[10] Q. Liu, J. Appl. Crystallogr. 27, 755 (1994).
[11] D. A. Hughes and N. Hansen, Acta Mater. 48, 2985 (2000).

[12] D. A. Hughes and N. Hansen, Philos. Mag. 83, 3871 (2003).

[13] S. Aubry and D. A. Hughes, Phys. Rev. B, 73, 224116 (2006).

[14] A. Godfrey and D. A. Hughes, Acta Mater. 48, 1897 (2000). 